UCRL-JC-123734

PREPRINT

CONF-9503244--1

\title{
Rheology of Planetary Ices
}

W.B. Durham, S.H. Kirby, and L.A. Stern

This paper was prepared for submittal to the Proceedings of the Solar System Ices Conference

27-30 March 1995, Toulouse, France

April 24, 1996

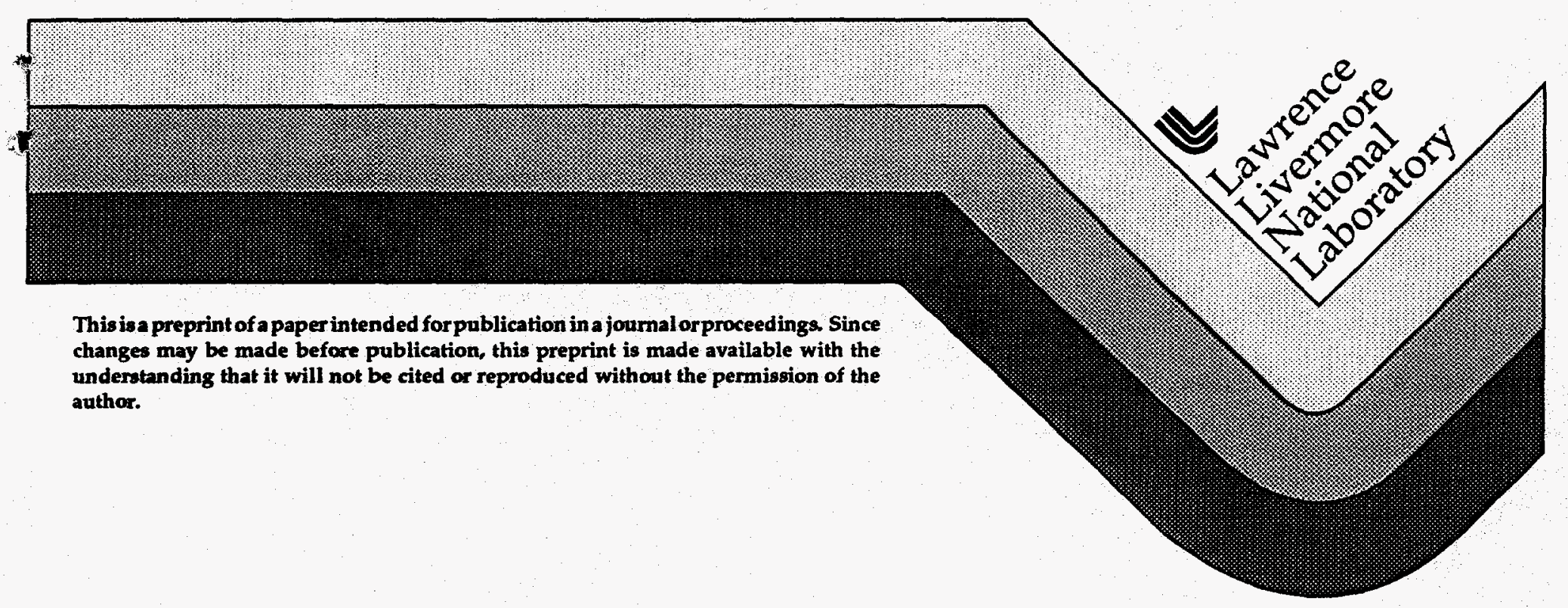




\section{DISCLAIMER}

This document was prepared as an account of work sponsored by an agency of the United States Government. Neither the United States Government nor the Liniversity of California nor any of their emplovees. makes any warranty. express or imptied. or assumes any legal liability or responsibility for the accuracy. completeness. or usefuiness of any information. apparatus. product, or process disclosed. or represents that its use would not infringe privately owned rights. Reference herein to any specific commercial products. process. or service by trade name, trademark. manufacturer. or otherwise, does not necessarity constitute or imply its endorsement. recommendation. or favoring by the Lited States Government or the University of California. The views and opinions of authors expressed herein do not necessariiy state or reflect those of the United States Government or the University of California. and shall not be used for advertisine or product endorsement purposes. 


\title{
Rheology of Planetary Ices
}

\author{
William B. Durham ${ }^{1}$, Stephen H. Kirby ${ }^{2}$, and Laura A. Stern ${ }^{2}$ \\ ${ }^{1}$ University of California, Lawrence Livermore National Laboratory \\ Livermore, CA 94550 \\ (510) $422-7046$ \\ ${ }^{2}$ United States Geological Survey, Menlo Park, CA 94025
}

\begin{abstract}
Laboratory measurements of the brittle and ductile behavior of several of the major icy planetary building materials have been made: water ice phases I through VI, ices in the ammonia-water system, mixtures of water ice plus particulates, and methane clathrate. All the materials measured thus far are sufficiently ductile that they can be expected to participate in convective motion in planetary interiors, and the quantitative rheological laws presented here provide constraints for models of evolutionary and present-day processes on icy moons of the solar system. Some unusual behavior is worth special note: ice III is several orders of magnitude less viscous than the other water ice phases. Ammonia dihydrate has a strength that is so temperature sensitive that, although it melts near $176 \mathrm{~K}$, it becomes as strong as ice I after a decrease in temperature of only $23 \mathrm{~K}$. Because of the extrapolation over many orders of magnitude in strain rate from the laboratory to planetary interiors, some of the material strength values calculated on the basis of the laboratory data may be only upper bounds on strength in the planetary setting.
\end{abstract}

\section{Introduction}

The brittle and ductile rheology of ices of water, ammonia, methane, and other volatiles, in combination with rock particles and each other, have a primary influence on the evolution and ongoing tectonics of icy moons of the outer solar system [Consolmagno and Lewis, 1976; Parmentier and Head, 1979; Kirk and Stevenson, 1987; Mueller and McKinnon, 1988]. Laboratory experiments help constrain the rheology of solar system ices. Standard experimental techniques can be used because the physical conditions under which most solar system ices exist 
are within reach of conventional rock mechanics testing machines, adapted to the low subsolidus temperatures of the materials in question. In fact, the mechanical properties of ices under planetary conditions are far better known than those of other earth materials under most geological conditions simply because the relevant temperatures and pressures are so much easier to reach and because planetary ices generally involve chemically simpler systems.

The low-temperature rheologies of the major icy planetary components (Figure 1) have been measured: water ice I [Durham et al., 1983; Kirby et al., 1987; Durham et al., 1992], ices II through VI [Echelmeyer and Kamb, 1986; Sotin et al., 1985; Sotin and Poirier, 1987; Durham et al., 1988, 1995], ammonia dihydrate [Durham et al., 1993], and methane clathrate [Durham and Kirby, 1990]. Important planetary mixtures have also been measured: ice I + hard particulates [Durham et al., 1992] and ice I + ammonia dihydrate [Durham et al., 1993]. Measurements have also been made in the brittle field, with application to the low-pressure, low-temperature region near planetary surfaces, on ice I [Beeman et al., 1988; Kirby et al., 1991], and on ice I + particulates [Durham et al., 1992].

The purpose of this review is to summarize the results of a decade-long experimental deformation program in our laboratories, and to provide some background in deformation physics in order to lend some appreciation to the application of these measurements to the planetary setting. It should be pointed out that limited data exist for several other ices, some of which are given in other chapters of this book. Strength measurements have been made directly on solid $\mathrm{CH}_{4}$, and indirectly on solid $\mathrm{N}_{2}$ [both are reviewed by Eluszkiewicz and Stevenson, 1990]. Data exist for the strength of $\mathrm{CO}_{2}$ at 1 atmosphere with application to the Martian surface [Clark and Mullin, 1976], on sulfate hydrates [Kargel, 1993], and on a number of more exotic HCON compounds.

\section{Background}

Flow laws. We will first review some of the basic principles of deformation experiments used in the interpretation of creep data. High-temperature creep is a general term comprising almost any and all of the of the myriad of volume-conservative deformation mechanisms that individually involve the motion of crystal-lattice or grain-boundary defects [see, e.g. Poirier, 1985]. Because of the large strains $(>1)$ usually encountered in planetary applications, our concern here is mainly with steady-state creep, which can be expressed as

$$
\dot{\varepsilon}=A \sigma^{n} \exp \left(-\frac{E^{*}+P V^{*}}{R T}\right)
$$


The flow law (1) is independent of the absolute value of strain $\varepsilon$, reflecting the independence of steady-state flow and deformation history. Although based to a certain extent in deformation physics, especially in the thermal activation term, the flow law is largely empirical. It reflects the concept that a sample under applied stress $\sigma$ responds by deforming at a strain rate $\dot{\varepsilon}$ and that the relationship between $\dot{\varepsilon}$ and $\sigma$ is influenced by environmental variables hydrostatic pressure $P$ and temperature $T$. Other environmental variables, such as grain size or chemical activity of components, can be added to the flow law if they are found to be relevant. The other symbols in (1) are the gas constant $R$ and four material constants: $A$, the pre-exponential; $n$, the stress exponent; $E^{*}$, the activation energy; and $V^{*}$, the activation volume. Different deformation mechanisms are expressed in terms of (1) with different sets of material constants.

It is important to understand that all thermally activated deformation mechanisms are functioning simultaneously if $T>0 \mathrm{~K}$. The flow law (1) is a satisfactory vehicle for expressing the combined operation of many mechanisms because under most conditions one mechanism will dominate to the point that all other mechanisms can be virtually ignored. The only reason it is even meaningful to discuss different mechanisms is because geology is unique among sciences concerned with deformation of materials in that the range of relevant strain rates is extremely broad; nearly twenty orders of magnitude separate strain rates in shear zones during seismic instabilities and those in the interiors of real or hypothesized mantle convection cells.

Most deformation experiments in the laboratory are, by necessity, conducted over a narrow range of relatively fast strain rates, roughly $10^{-4}$ to $10^{-7} \mathrm{~s}^{-1}$. There are several consequences of sampling a narrow portion of a field where several mechanisms of the form (1) operate simultaneously (Figure 2). One consequence is simply that the mechanism that dominates (i.e. is observed) at laboratory strain rates will not necessarily dominate at rates orders of magnitude slower. It can be seen in the figure that extrapolation of the laboratory measurements to lower strain rates provides at least an upper bound on strength. Another consequence is that certain effects viewed in the laboratory are illusory. In particular, it is not uncommon to observe that as temperature is raised, the value of the stress exponent $n$ tends to fall. Often overlooked is the fact that as temperature is increased the operator tends to reduce the magnitude of stress in order to prevent strain rates from proceeding too fast to be measured. As shown in Figure 2, it is important to add to this observation the qualification that strain rate is held constant. If temperature is raised at fixed stress (which is difficult in the laboratory, but no less relevant to planetary interiors), a mechanism of higher $n$ is favored.

Creep experiments. To measure plastic deformation, that is, volume conservative flow, it is necessary to confine the test sample in order to suppress deformation facilitated by microfracture. Creep apparatuses used in geology thus often feature fairly massive pressure vessels, and in the case of water ices, such a pressure vessel also provides the means of accessing the stability fields 
of relevant high-pressure phases. The creep apparatus also must feature the means for measuring the dependent variable and controlling the other independent variables. The apparatus that produced most of the results in this paper [Heard et al., 1990] has a 1-GPa pressure limit, a cylindrical geometry, and takes a polycrystalline sample of relatively large volume (4 to $\left.30 \mathrm{~cm}^{3}\right)$. Temperature is controlled by a cold bath surrounding the vessel, and the other independent variable, strain rate, is controlled by a sliding piston that compresses the sample against a static force gauge inside the vessel. Figure 3 shows the principal features of a deformation curve. The value of stress required to keep the sample deforming at a constant rate usually reaches a point after 5 to $10 \%$ of strain where it ceases evolving with strain. It is this value that we take for the value of $\sigma$ in Equation (1).

\section{Flow of Planetary Ices}

Brittle and ductile flow of water ice I. Ice I [Durham et al., 1983; Kirby et al., 1987; Durham et al., 1992] is the most extensively measured of all planetary ices. An extensive literature also exists for ice deformed under conditions appropriate to terrestrial glaciers and sea ice [Weertman, 1983]. Over the laboratory range of strain rates ice I exhibits three flow regimes (Figure 4, Table 1). From overlap with the observations from the glaciological literature, it appears that flow in the high-temperature regime $(T>240 \mathrm{~K}$ ) is controlled by the presence of grain boundaries in the ice, but the nature of this control is still poorly understood. Observations of nearly equantshaped grains in even highly strained samples from both the intermediate- and low-temperature regimes indicate recrystallization is active, but the cause of the break in mechanism near $T=195$ $\mathrm{K}$ is still unclear.

The data in Figure 4 derive from polycrystalline samples with large enough grain size $(>0.25$ $\mathrm{mm}$ ) that, except perhaps for the high-temperature regime, the strength is not influenced by changes in grain size [Durham et al., 1992]. Recent measurements on much finer grained ice suggest the same may not be true when grain size falls below about $0.050 \mathrm{~mm}$ [Durham et al., 1994; Goldsby and Kohlstedt, 1995]. The former study shows weakening of about one order of magnitude in viscosity in fine grained ice as compared to that in Figure 4. Goldsby and Kohlstedt [1995] found that strain rate increases as (grain diameter) ${ }^{-2}$. This finding is quite significant because it confirms theoretical predictions (e.g. Frost and Ashby, 1982) that the strength of ice I at very low strain rates (i.e. planetary) will be governed by grain-size-dependent rheology even if ice grains are a more realistic 1 to $10 \mathrm{~mm}$ grain size.

Flow of ice $I+$ dispersed particulates. Based on analogy to metals, it has been suggested that mixtures of ice and small amounts of rock may strengthen ice sufficiently to alter models of interior processes or help explain retention of surface topography on some of the moons [see references in Durham et al., 1992]. Laboratory measurements (Figure 5) suggest otherwise. The addition of hard particulates does strengthen ice $I$, but only if added in sufficient volume to hinder flow macroscopically. In the intermediate and low temperature flow regimes of ice I there 
seems to be no dependence on the size (from 1 to $150 \mu \mathrm{m}$ ) or composition (SiC, calcite, quartz) of the particulates, only their total volume. The hindrance that appears at volume fractions above $10 \%$ is more pronounced than can be explained by geometric lengthening of flow paths, and may be related to viscous drag of ice around the particles.

Brittle behavior of ice. Ice becomes brittle, i.e. prone to sudden strength loss, as pressure or temperature decreases, or as stress or imposed strain rate increases. The brittle regime most closely resembles the near-surface planetary environment where faulting, movement on existing faults, and slope collapse can occur. Like most rocks in the brittle field, ice has a strength at failure that is nearly independent of temperature or strain rate, but strongly dependent on confining pressure, at least in the range $0<P<50 \mathrm{MPa}$. Figure 6 shows the strength at failure of ice I at $77 \mathrm{~K}$ as a function of confining pressure [Durham et al., 1983; Kirby et al., 1991]. The disappearance of the pressure dependence above $P=50 \mathrm{MPa}$ is profoundly unusual. The mechanism by which ice I fails at elevated confining pressure we call transformational faulting because it involves limited nucleation of ice II just prior to failure, and is apparently triggered by stress interaction of ice II microinclusions under high shear stress. Transformational faulting can conceivably occur locally on icy moons during large impacts [Kirby and Durham, 1991], but is most interesting because of its potential relationship to the mechanism of deep terrestrial earthquakes [Kirby et al., 1991, 1995].

An icy crust that has already faulted will have a lower strength than unfaulted ice. Based on measurements of laboratory samples at $T \leq 143 \mathrm{~K}$, which probably give an upper bound to strength of a faulted icy crust because of scaling effects, the shear strength of a preexisting fault is approximately $30 \%$ of the normal stress on the fault [Beeman et al., 1988]. Above approximately $10 \mathrm{MPa}$ normal stress, the frictional strength of machined flat surfaces is not significantly different from that of a fault formed by fracturing intact material. Above $250 \mathrm{MPa}$, faulted and intact material have almost the same strength, suggesting that friction at high pressures may also be limited by the ice I $\rightarrow$ II transformation.

Flow of ices in the ammonia-water system. Cosmochemical considerations of the solar nebula suggest that where temperatures were cool enough to allow condensation of $\mathrm{NH}_{3}$, approximately at the orbit of Saturn and beyond, ammonia/water ratios of approximately 0.15 might be expected [Lewis, 1972; Prinn and Fegley, 1981]. The appearance of extensive resurfacing on moons of Saturn and Uranus [Schenk, this volume] also argues for the presence of condensed ammonia, because peritectic melting occurs on the water-rich side of the ammoniawater system at $0.1 \mathrm{MPa}$ and $176 \mathrm{~K}$ (increasing to about $180 \mathrm{~K}$ at $100 \mathrm{MPa}$ ). Two equilibrium phases exist in the water-rich end of the system at mole fraction $\mathrm{NH}_{3}<0.33$ : water ice $\mathrm{I}$ and ammonia dihydrate, $\mathrm{NH}_{3} \cdot 2 \mathrm{H}_{2} \mathrm{O}$. Durham et al. [1993] measured the rheology of mixtures of these two phases to low temperatures and pressures to $100 \mathrm{MPa}$ (Figure 7). The results suggest that if dihydrate/water mixtures are to act as a soft material on planetary surfaces, temperatures must be chosen very carefully. At temperatures just below the $176 \mathrm{~K}$ peritectic, ice I and dihydrate differ in strength by a factor of 5 , or by a factor of $10^{4}$ if stated in terms of a viscosity 
contrast. However, the strength of pure dihydrate is extraordinarily temperature sensitive, and reaches that of pure ice I at about $143 \mathrm{~K}$. The flow laws of water/dihydrate mixtures show a systematic variation from that of pure ice I to that of pure dihydrate. No deformation experiments have been successfully performed below $140 \mathrm{~K}$ to determine if dihydrate actually becomes stronger than water. All such attempts terminated with brittle failure of the samples, perhaps because of the presence of small amounts of ice I causing a recurrence of the shear instability discussed above. Ammonia monohydrate, $\mathrm{NH}_{3} \bullet \mathrm{H}_{2} \mathrm{O}$, formed metastably in a few experiments [Durham et al., 1993], and looks qualitatively to be even weaker than dihydrate.

Flow of methane clathrate. Durham and Kirby [1990] have reported very preliminary results for the strength of methane clathrate, $\mathrm{CH}_{4} \bullet 6 \mathrm{H}_{2} \mathrm{O}$. They found that at $T=160$ and $180 \mathrm{~K}$ and $P=$ $50 \mathrm{MPa}$, methane clathrate is slightly weaker but comparable in strength to water ice I. The creep of clathrate showed an extended period of strain hardening, quite different than the type of behavior (Figure 3) seen for most other ices. Further research is currently underway.

Flow of high-pressure water phases II, III, V, and VI. These phases are assumed to be the primary building materials of the giant icy moons of the outer solar system, so understanding of the evolution of these moons requires basic understanding of their physical properties. The rheologies of ices II and III have been measured by Durham et al. [1988] and Echelmeyer and Kamb [1986]; rheologies of ices V and VI by Durham et al. [1988, 1995], Sotin et al. [1985], and Sotin and Poirier [1987]. They are compared to the rheology of ice I in Figure 8. The field of each phase in Figure 8 covers the same two orders of magnitude in strain rate vertically and horizontally covers the range of temperature explored experimentally. Since the phases do not all coexist at the same pressure, rheologies for individual phases are shown at representative pressures. If phases are to be compared directly, pressure dependence (Table 1) must be applied. Thus ice II is the hardest phase at laboratory conditions, but the contrast between phases I, II, V, and VI is not pronounced. Ices V and VI at lab conditions have distinct rheologies in terms of flow parameters (Table 1), but have overlapping strengths at laboratory conditions. Standing out in sharp contrast is the weakness of ice III (Figure 8), showing a viscosity contrast of several orders of magnitude with all its neighbors. If this contrast remains present at planetary strain rates (see below), it may require special consideration in models of mantle convection. Although its stability field is rather small (Figure 1), the location of that field falls along many suggested planetary temperature-depth profiles. Furthermore, ice III demonstrates in the laboratory a rather profound metastability in the warm temperature region of ice II.

It should be mentioned that there is not good agreement between the two groups listed above regarding the strength of ice V. The possible causes are discussed in Durham et al. [1988, 1995]. We believe that the constants given in Table 1 are more likely to be correct because they are based on more measurements and were made using a more direct method.

There is a possibility that mechanical weakness observed in ice II formed by transformation from I under nonhydrostatic stress may be the result of a decreased grain size in the II phase [Stern et al., 1994]. Partially transformed samples of ice I contain regions of fine-grained ice II 
showing localized strain, indicating that fine-grained ice II deforms faster than ice I under the

same conditions of nonhydrostatic stress. Ordinarily II is somewhat stronger than I (Figure 8). Interestingly, the microstructure of the ice II in those samples shows a similarity to that of ice I deformed in the fine-grained regime [Durham et al., 1994].

\section{Conclusions}

The icy building materials that comprise the moons of the outer solar system, to the extent they have been measured (Table 1), generally present no impediment to models of planetary evolution that involve convective motion within icy layers. Worthy of special note are (1) the lack of profound strengthening of ice $I$ in the presence of low $(<30 \%)$ volume concentrations of impurities, (2) the very low strength of ice III, which may never allow it to support a very large temperature gradient, (3) the probability that ice I and perhaps II deformed in the grain-sizedependent regime may be weaker than expressed by the flow constants in Table 1, and (4) the very high temperature sensitivity of the strength of ammonia dihydrate, which provides planetologists with a rather restricted temperature range within which dihydrate may behave plastically on planetary surfaces.

\section{Acknowledgments}

The work would not have been possible without the participation of Hugh Heard, who died several years after our research program began. The technical assistance of many individuals, most notably M. A. Daley and C. O. Boro, has been invaluable. Financial support by NASA throughout this project is gratefully acknowledged, as is the periodic support to one of us (WBD) from the German Alexander von Humboldt Foundation. This work was performed under the auspices of the U. S. Department of Energy by the Lawrence Livermore National Laboratory under contract W-7405ENG-48. 


\section{References}

Beeman, M. L., W. B. Durham, and S. H. Kirby, Friction of ice, J. Geophys. Res., 93, 7625-7633, 1988.

Consolmagno, G. J. and J. S. Lewis, Structural and thermal models of icy galilean satellites, in Jupiter, T. Gehrels ed., Univ. of Arizona Press, 1035-1051, 1976.

Clark, B. R. and R. P. Mullin, Martian glaciation and the flow of solid $\mathrm{CO}_{2}$, Icarus, 27, 215-228, 1976.

Durham, W. B. and S. H. Kirby, Planetary ices: A comparison of rheologies at $T<200 \mathrm{~K}$ (abstract), Proc. 21st Lunar Planet. Sci. Conf., 305-306, 1990.

Durham, W. B., H. C. Heard, and S. H. Kirby, Experimental deformation of polycrystalline $\mathrm{H}_{2} \mathrm{O}$ ice at high pressure and low temperature: preliminary results, J. Geophys. Res., 88, B377-B392, 1983.

Durham, W. B., S. H. Kirby, H. C. Heard, L. A. Stern, and C. O. Boro, Water ice phases II, III, and V: Plastic deformation and phase relationships, J. Geophys. Res., 93, 10191-10208, 1988.

Durham, W. B., S. H. Kirby, and L. A. Stern, Effects of dispersed particulates on the rheology of water ice at planetary conditions, J. Geophys. Res., 97, 20883-20897, 1992.

Durham, W. B., S. H. Kirby, and L. A. Stern, Flow of ices in the ammonia-water system, J. Geophys. Res., 98, 17667-17682, 1993.

Durham, W. B., L. A. Stern, and S. H. Kirby, Grain size dependent creep of ice I at low temperature: preliminary results (abstract), EOS Trans. Am. Geophys. Un., 75, 635, 1994.

Durham, W. B., L. A. Stern, and S. H. Kirby, Rheology of water ices V and VI, J. Geophys. Res., submitted, 1995.

Echelmeyer, K. and B. Kamb, Rheology of ice II and ice III from high-pressure extrusion, Geophys. Res. Lett., 13, 693-696, 1986.

Eluszkiewicz, J. and D. J. Stevenson, Rheology of solid methane and nitrogen: Applications to Triton, Geophys. Res. Lett., 17, 1753-1756, 1990. 
Goldsby, D. L. and D. L. Kohlstedt, The transition from dislocation to diffusion creep in ice, Proc. Lunar Planet. Sci. Conf. 26th, 473-474, 1995.

Heard, H. C., W. B. Durham, C. O. Boro, and S. H. Kirby, A triaxial deformation apparatus for service at $77 \leq \mathrm{T} \leq 273 \mathrm{~K}$, in The Brittle-Ductile Transition in Rocks, Geophysical Monograph 56, ed. by A. G. Duba, W. B. Durham, J. W. Handin, and H. F. Wang, American Geophysical Union, Washington, D. C. pp. 225-228, 1990.

Kirby, S. H. and W. B. Durham, Deep moonquakes on Ganymede? (abstract), Proc. 22nd Lunar Planet. Sci. Conf., 719-720, 1991.

Kirby, S. H., W. B. Durham, M. L. Beeman, H. C. Heard, and M. A. Daley, Inelastic properties of ice $\mathrm{I}_{\mathrm{h}}$ at low temperatures and high pressures, J. Physique, 48, supplement (VIIth Symposium on the Physics and Chemistry of Ice), 227-232, 1987.

Kirby, S. H., W. B. Durham, and L. A. Stern, Mantle phase changes and deep-earthquake faulting in subducting lithosphere, Science, 252, 216-225, 1991.

Kirby, S. H., S. Stein, E. Okal, and D. Rubie, Deep earthquakes and metastable mantle phase transformations in subducting oceanic lithosphere, Reviews of Geophysics (invited), submitted, 1995.

Kirk R. L. and D. J. Stevenson, Thermal evolution of a differentiated Ganymede and implications for surface features, Icarus, 69, 91-134, 1987.

Lewis, J. S., Low temperature condensation from the solar nebula, Icarus, 16, 241-252, 1972.

Mueller, S. and W. B. McKinnon, Three-layered models of Ganymede and Callisto: Compositions, structures, and Aspects of evolution, Icarus, 76, 437-464, 1988.

Parmentier, E. M. and J. W. Head, Internal processes affecting surfaces of low-density satellites: Ganymede and Callisto, J. Geophys. Res., 84, 6263-6276, 1979.

Poirier, J.-P., Creep of Crystals, Cambridge University Press, Cambridge, 260 pp., 1985.

Prinn, R. G. and B. Fegley, Jr., Kinetic inhibition of $\mathrm{CO}$ and $\mathrm{N}_{2}$ reduction in circumplanetary nebulae: implications for satellite composition, Astrophys. J., 249, 308-317, 1981. 
Sotin, C., P. Gillet, and J.-P. Poirier, Creep of high-pressure ice VI, in Ices in the Solar System, J. Klinger et al., ed., D. Reidel Publishing Co., Dordrecht, Holland, pp. 109-118, 1985.

Sotin, C. and J.-P. Poirier, Viscosity of ice V, J. Physique, 48 supplement (VIIth Symposium on the Physics and Chemistry of Ice), 233-238, 1987.

Stern, L. A., S. H. Kirby, and W. B. Durham, Textural observations of $\mathrm{H}_{2} \mathrm{O}$ ice II nucleation and growth processes during phase transformations under hydrostatic and nonhydrostatic stress (abstract), EOS Trans. Am. Geophys. Un., 75, 635, 1994.

Weertman, J., Creep deformation of ice, in Annu. Rev. Earth Planet. Sci., 215-240, 1983. 
Figure 1. The phase diagram of water and the melting curve of icy compounds of planetary importance.

Figure 2. Rheology of an imaginary (poly)crystalline solid demonstrating the relationship between perceived and real behavior. Shown are three flow regimes described mathematically by the flow law (Equation 1) and three different sets of material constants. Here pressure is fixed, and constants $E^{*}$ and $V^{*}$ are combined into a single parameter $Q=E^{*}+P V^{*}$. Nine long-dashed lines show the flow in each of the three regimes at each of three temperatures. The strain rate $\dot{\varepsilon}$ that results from the application of a differential stress $\sigma$ at, say, temperature $T_{2}$ will be the sum of the contributions from each mechanism. The vertical scale is logarithmic, so the trace of flow at $T_{2}$ for all $\sigma$ will be along the long solid line, with slight rounding of the knees at the point of mechanism "change." The short dashed lines thus delimit the regions of domination of the three mechanisms, which are labeled by their $n$ values. The vertical axis represents the range of geologically interesting strain rates, while the hatched region shows the limit of most laboratory experience. Thus the observation that $\dot{\varepsilon} \propto \sigma^{4}$ in the lab may belie the existence of a mechanism of lower $n$ that will clearly dominate at much lower strain rate. Note also that if temperature is raised from $T_{1}$ to $T_{3}$, the experimentalist will observe a change in $n$ from 9 to 4 because of the requirement that he/she work within a realistic laboratory time frame. Unless the experimentalist is aware that he/she has lowered $\sigma$ in order to stay within the laboratory band, a false conclusion may result.

Figure 3. Idealized record of a constant strain rate compression test. The sample first responds elastically, until the load curve first bends (at the yield point). The strength maximum is most pronounced in ice I and ice with small concentrations of particulates. With continued strain, the load eventually reaches a constant value. This is the value for which the steady-state flow law (Equation 1) applies. Most planetary ices, with the exception of methane clathrate, display such behavior.

Figure 4. Flow of polycrystalline ice I at a fixed pressure of $50 \mathrm{MPa}$. Curves represent estimated fits to approximately 150 data points at four different fixed values of strain rate, as labeled. Dashed lines delimit the fields of the three different deformation mechanisms, in the style of Figure 2. Taken from Durham et al. [1992].

Figure 5. Flow of ice I plus silicate sand in varying volume concentrations $\phi$ at fixed pressure 50 MPa. Sand grain size is $0.15 \mathrm{~mm}$. Very little change occurs with variation in hard particulate size or composition. Dashed lines are the pure ice I flow from Figure 4. The "Shear Instability Limit" is set by the anomalous failure of ice I (e.g. Figure 6). Taken from Durham et al. [1992]. 
Figure 6. Failure strength of ice I at $T=77 \mathrm{~K}$. Ice I displays classic brittle behavior at low pressure, with strength increasing sharply with pressure. The pressure independent region results from the process of transformation faulting (see text).

Figure 7. Flow laws for ice mixtures in the water-rich end of the ammonia-water system at fixed strain rate $3.5 \times 10^{-6} \mathrm{~s}^{-1}$ and pressure $50 \mathrm{MPa}$. Curves are estimated fits to approximately 75 individual measurements. $\mathrm{x}_{\mathrm{NH} 3}$ is the mole fraction ammonia; $\mathrm{x}_{\mathrm{NH} 3}=0.33$ is pure ammonia dihydrate $\left(\mathrm{NH}_{3} \cdot 2 \mathrm{H}_{2} \mathrm{O}\right), \mathrm{x}_{\mathrm{NH} 3}=0.00$ is pure ice $\mathrm{I}$, and compositions in between are physical mixtures of the two end-member phases. The point on the lower horizontal axis labeled "melt" corresponds to the $176 \mathrm{~K}$ peritectic temperature where liquid first forms in mixtures of $x_{\mathrm{NH}_{3}} \leq 0.33$. Remarkable is the very strong temperature dependence of the strength of pure dihydrate. Although it is far weaker than ice I just below melting, it becomes as strong as ice at $143 \mathrm{~K}$. From Durham et al. [1993].

Figure 8. Comparison of the flow laws for ices I, II, III, V, and VI. Flow laws are represented as boxes bounded laterally by the experimental temperature range investigated and vertically by lines of constant strain rate. The same two strain rates apply to all fields, so it can be seen immediately, for example, that ice $\mathrm{V}$, which has a narrow vertical extent, has the highest value of $n$ (Table 1). Comparisons are made at values of fixed pressure representative for each phase. Note the extreme weakness of ice III and that the measured field of ice III extends to temperatures well below its field of phase stability. 
Flow constants.

$$
\dot{\varepsilon}=A \sigma^{n} \exp \left(-\frac{E^{*}+P V^{*}}{R T}\right)
$$

\begin{tabular}{|c|c|c|c|c|c|c|}
\hline & Material & $\begin{array}{c}\log \mathrm{A} \\
\left(\mathrm{MPa}^{-n} \mathrm{~S}^{-1}\right) \\
\end{array}$ & $n$ & $\begin{array}{c}E^{*} \\
(\mathrm{~kJ} / \mathrm{mole}) \\
\end{array}$ & $\begin{array}{c}V^{*} \\
\left(\mathrm{~cm}^{3 / \text { mole })}\right. \\
\end{array}$ & ref \\
\hline Ice I & $240-258 \mathrm{~K}$ & $11.8 \pm 0.4$ & $4 \pm 0.6$ & $91 \pm 2$ & a & Durham et al., 1992 \\
\hline Ice I & $195-240 \mathrm{~K}$ & $5.1 \pm 0.03$ & $4 \pm 0.1$ & $61 \pm 2$ & $13 \pm 3$ & $"$ \\
\hline Ice I & $<195 \mathrm{~K}$ & -1.4 & 5.6 & 43 & a & $"$ \\
\hline Ice II, & $220-250 \mathrm{~K}$ & $11.7 \pm 1.6$ & $5.2 \pm 0.3$ & $98 \pm 8$ & $4 \pm 4$ & Durham et al., 1988 \\
\hline Ice II & $<220 \mathrm{~K}$ & $1.84 \pm 0.27$ & $5.3 \pm 0.1$ & $55 \pm 2$ & $7 \pm 1$ & $"$ \\
\hline Ice III, & $230-250 \mathrm{~K}$ & $26.4 \pm 4.8$ & $4.3 \pm 1.2$ & $151 \pm 21$ & a & $"$ \\
\hline Ice III, & $<230 \mathrm{~K}$ & $13.3 \pm 1.5$ & $6.3 \pm 0.4$ & $103 \pm 8$ & a & $"$ \\
\hline Ice $\mathrm{V}$ & & 23.0 & $6.0 \pm 0.7$ & $136 \pm 38$ & $29 \pm 8$ & Durham et al., 1995 \\
\hline Ice VI ( & $\mathrm{T}<250 \mathrm{~K})$ & 6.7 & $4.5 \pm 0.2$ & $66 \pm 4$ & $11 \pm 6$ & $"$ \\
\hline Ice VI ( & $\Gamma>250 \mathrm{~K})$ & $(6.7)^{b}$ & $(4.5)^{b}$ & $110 \pm 20$ & $(11)^{b}$ & $"$ \\
\hline \multicolumn{3}{|c|}{ Ice I + hard particulatesc } & & & & Durham et al., 1992 \\
\hline \multicolumn{2}{|c|}{$\mathrm{NH}_{3} \cdot 2 \mathrm{H}_{2} \mathrm{O}$} & 21.55 & 5.81 & 107.5 & a & Durham et al., 1993 \\
\hline \multicolumn{3}{|c|}{$\mathrm{CH}_{4} \cdot 6 \mathrm{H}_{2} \mathrm{O}^{d}$} & & & & urham and Kirby, 1990 \\
\hline
\end{tabular}

aNot determined. Value of $E^{*}$ is thus activation enthalpy at $P=50 \mathrm{MPa}$ for ice $\mathrm{I}$ and $\mathrm{NH}_{3} \cdot 2 \mathrm{H}_{2} \mathrm{O}$, at $P=250 \mathrm{MPa}$ for ice $\mathrm{III}$. bEstimated based on ice VI results for $T<250 \mathrm{~K}$

cResults shown graphically (Figure 5)

dPreliminary results only: strength comparable to that of ice I at $T=160,180 \mathrm{~K}$ 


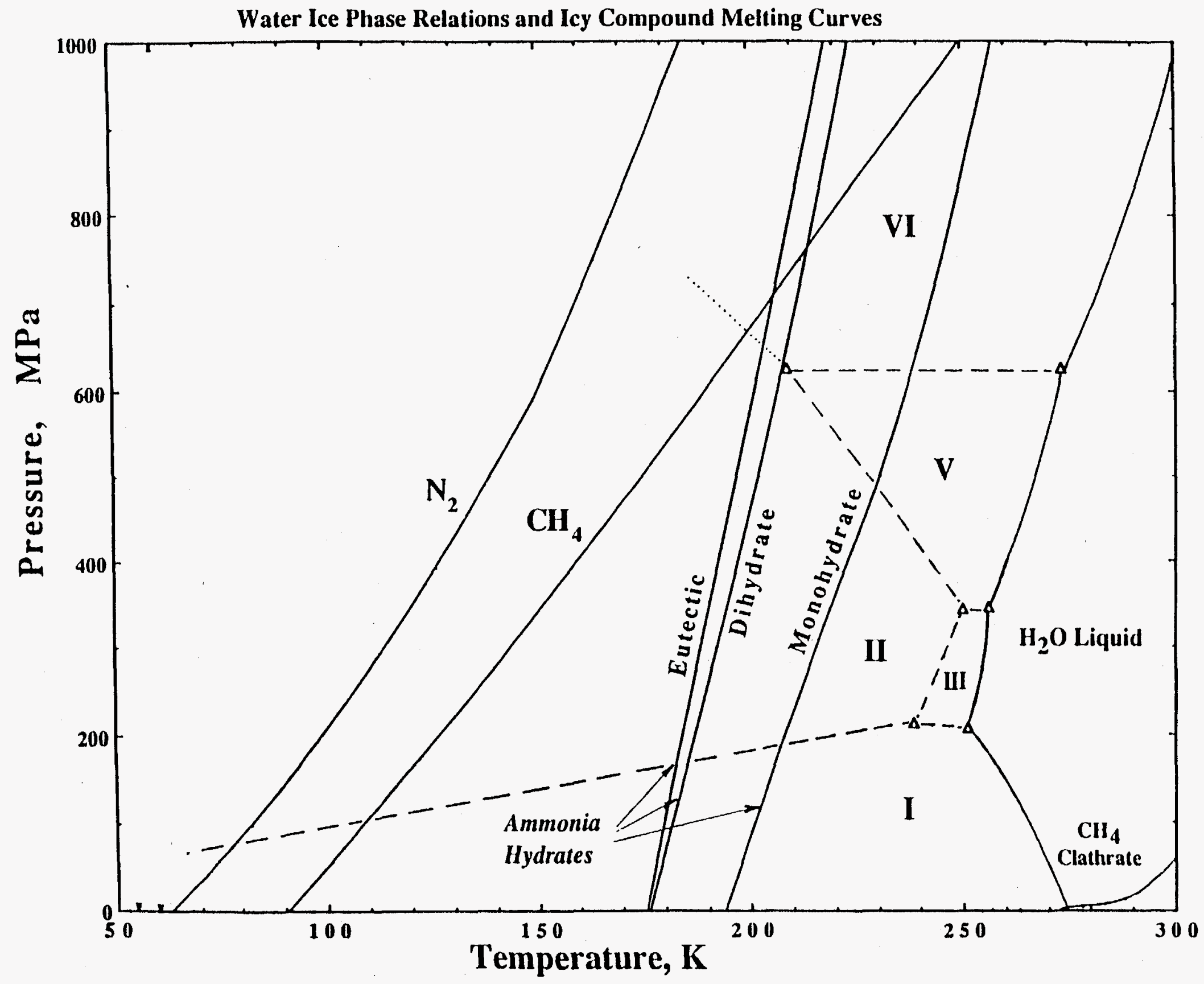




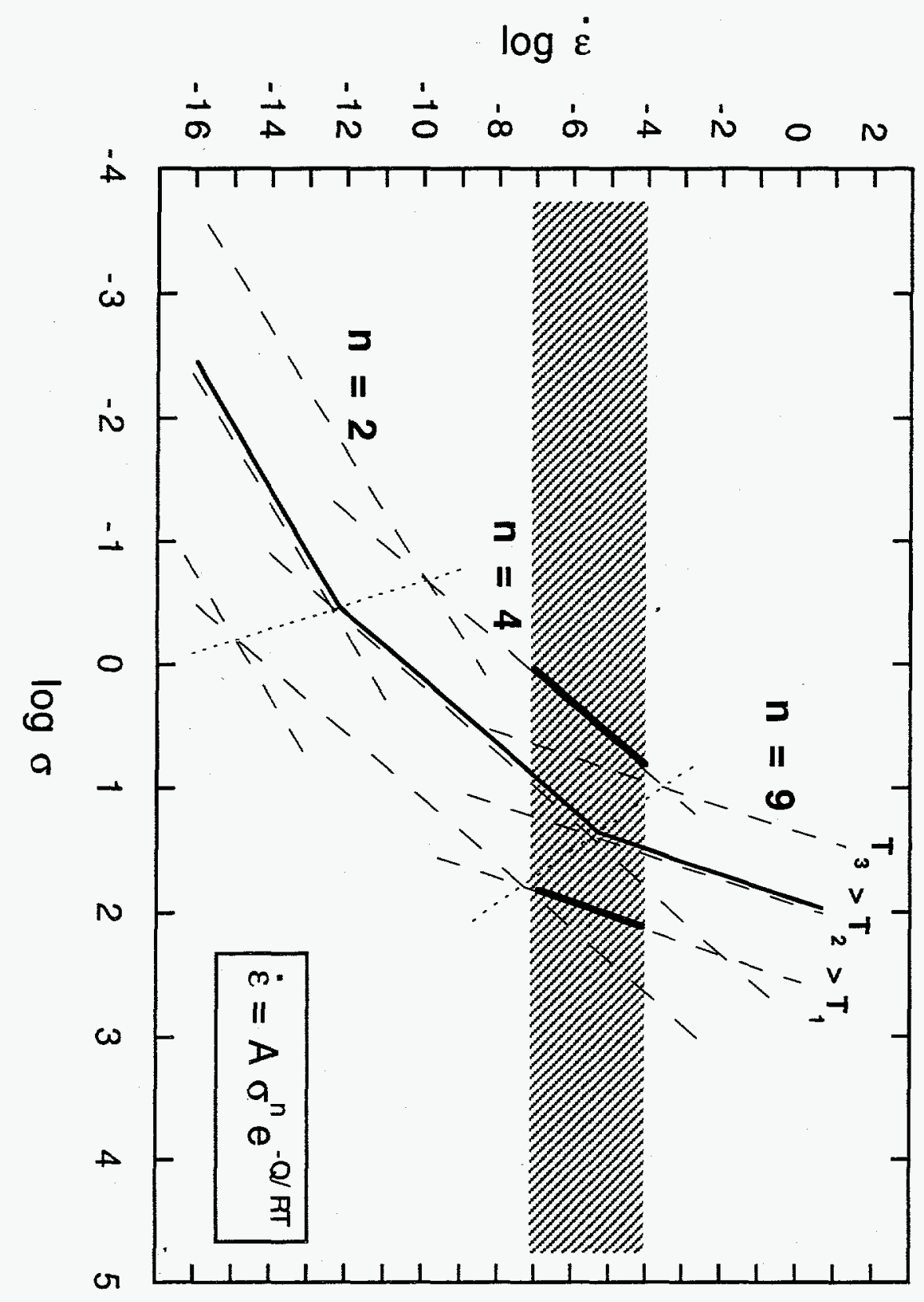

FIGURE2 


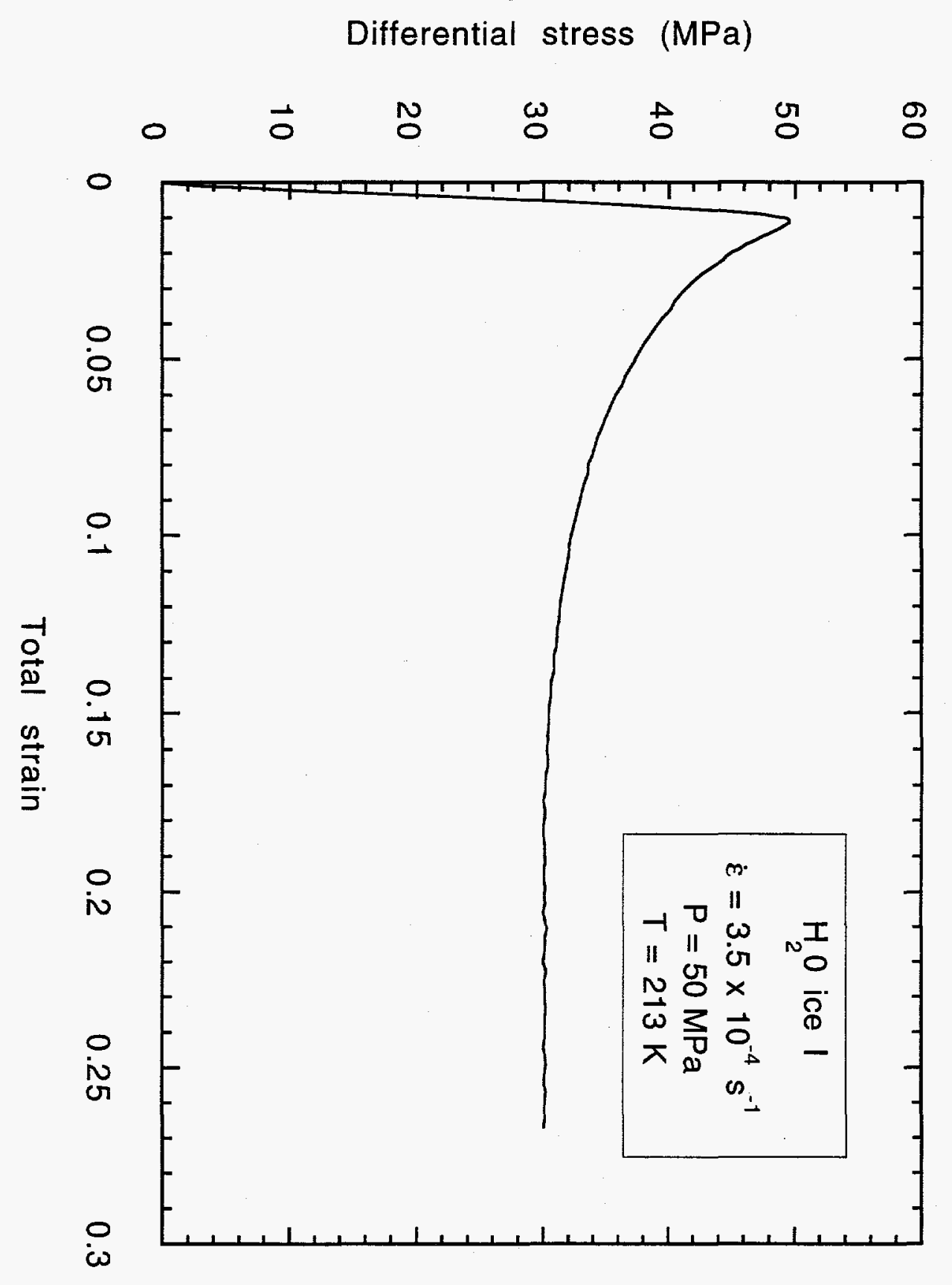

FIGURE 3 


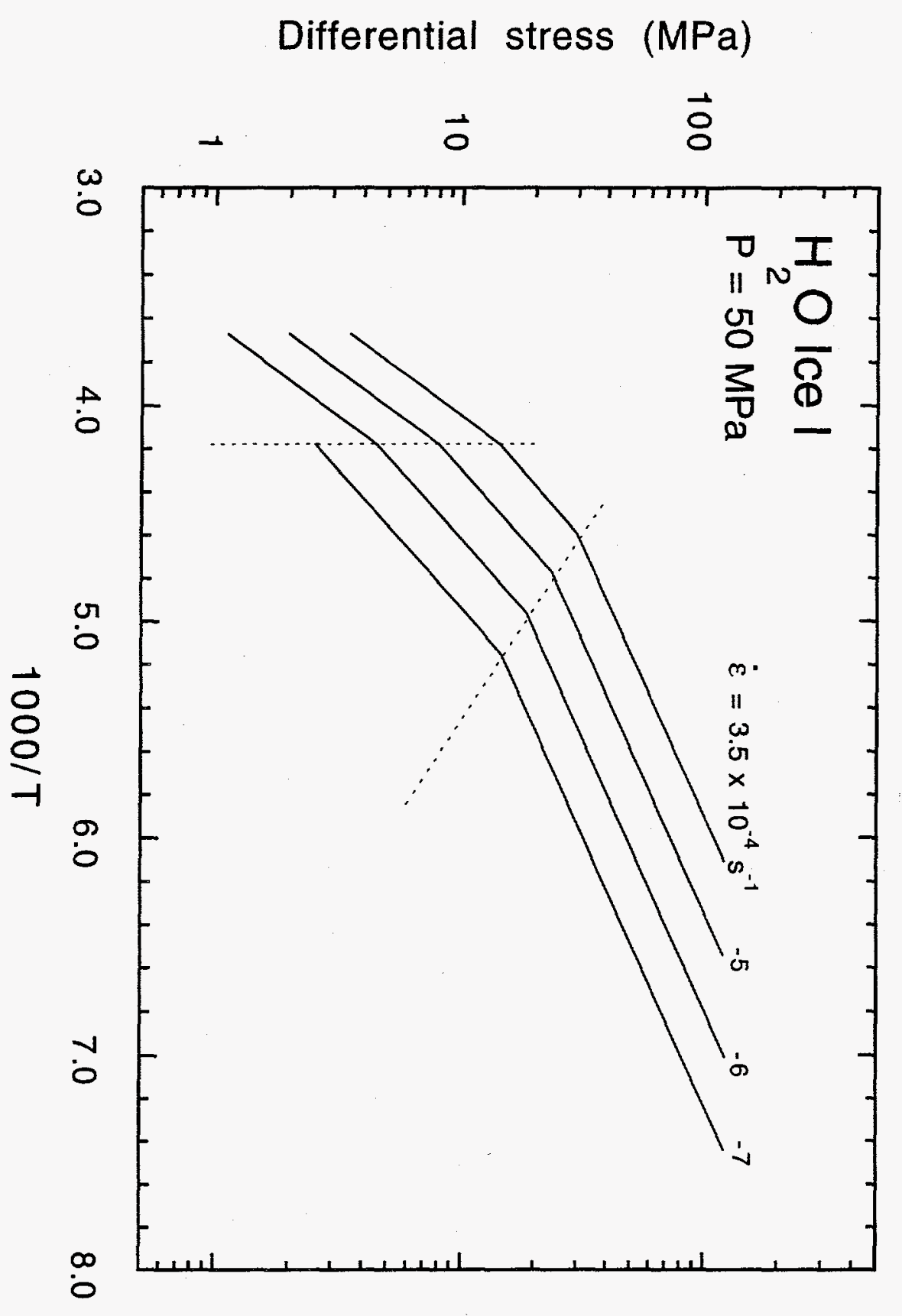

FIGURE 4 


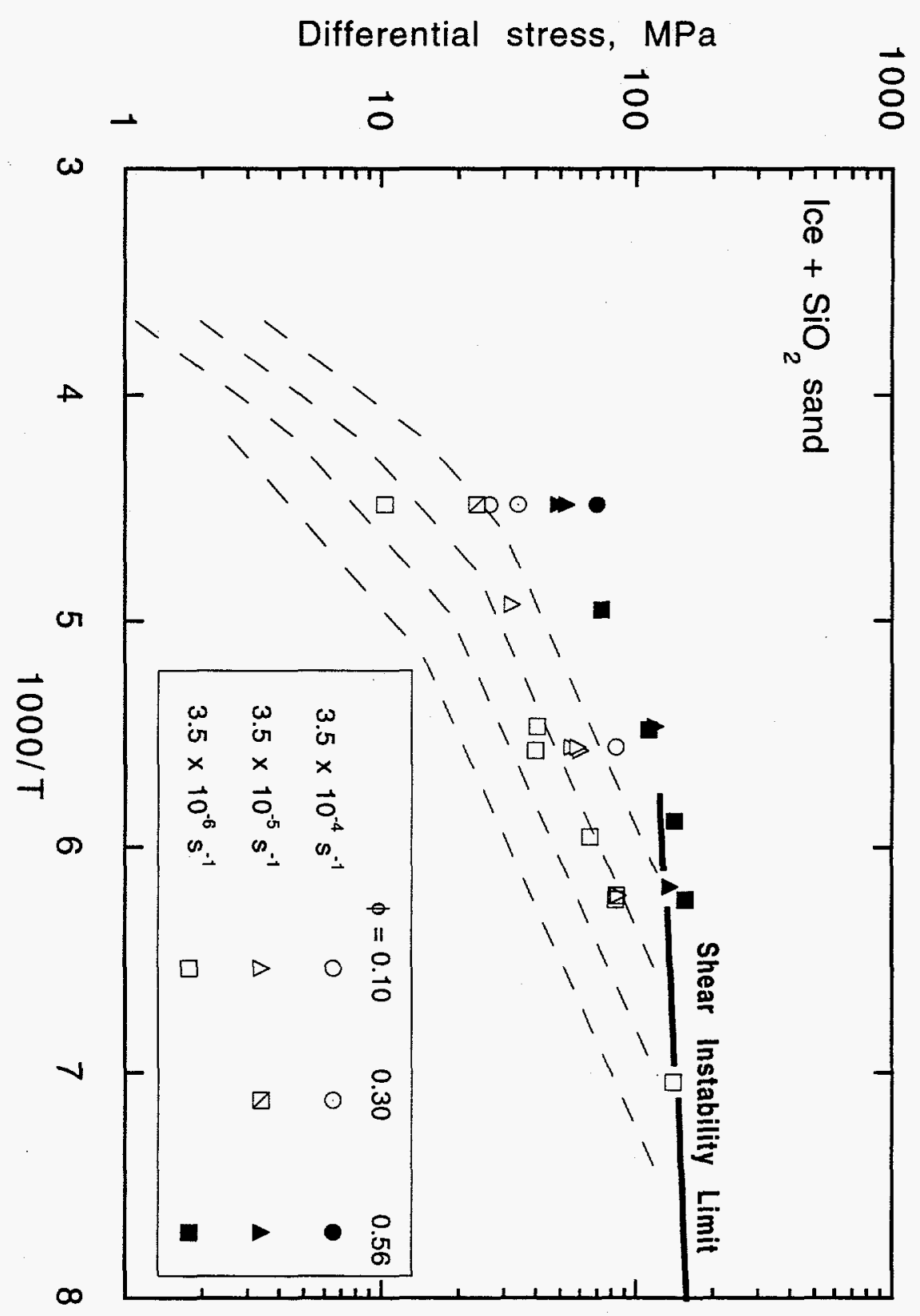

FIGURE 5 
Differential stress at failure (MPa)

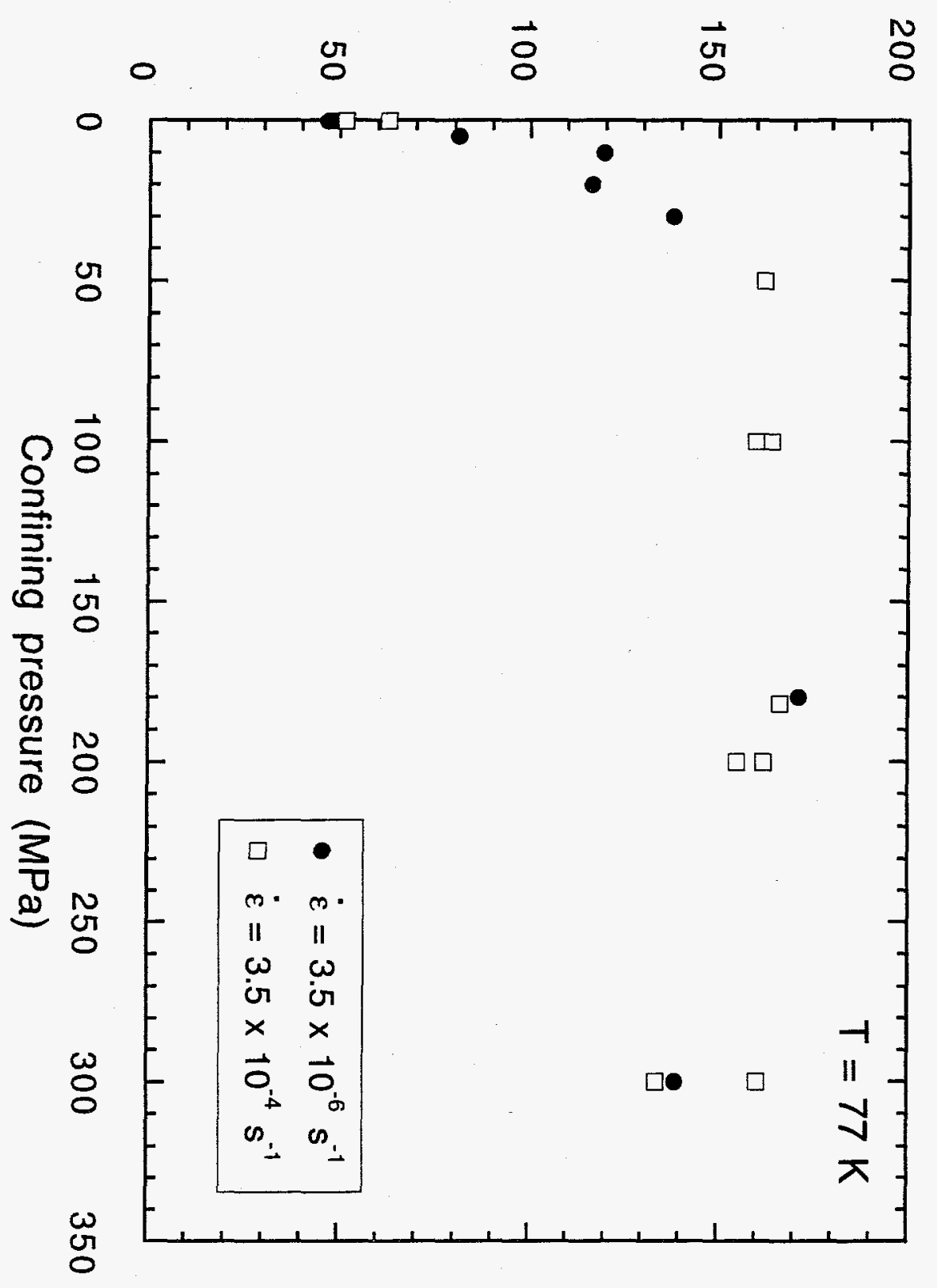

FIGURE 6 


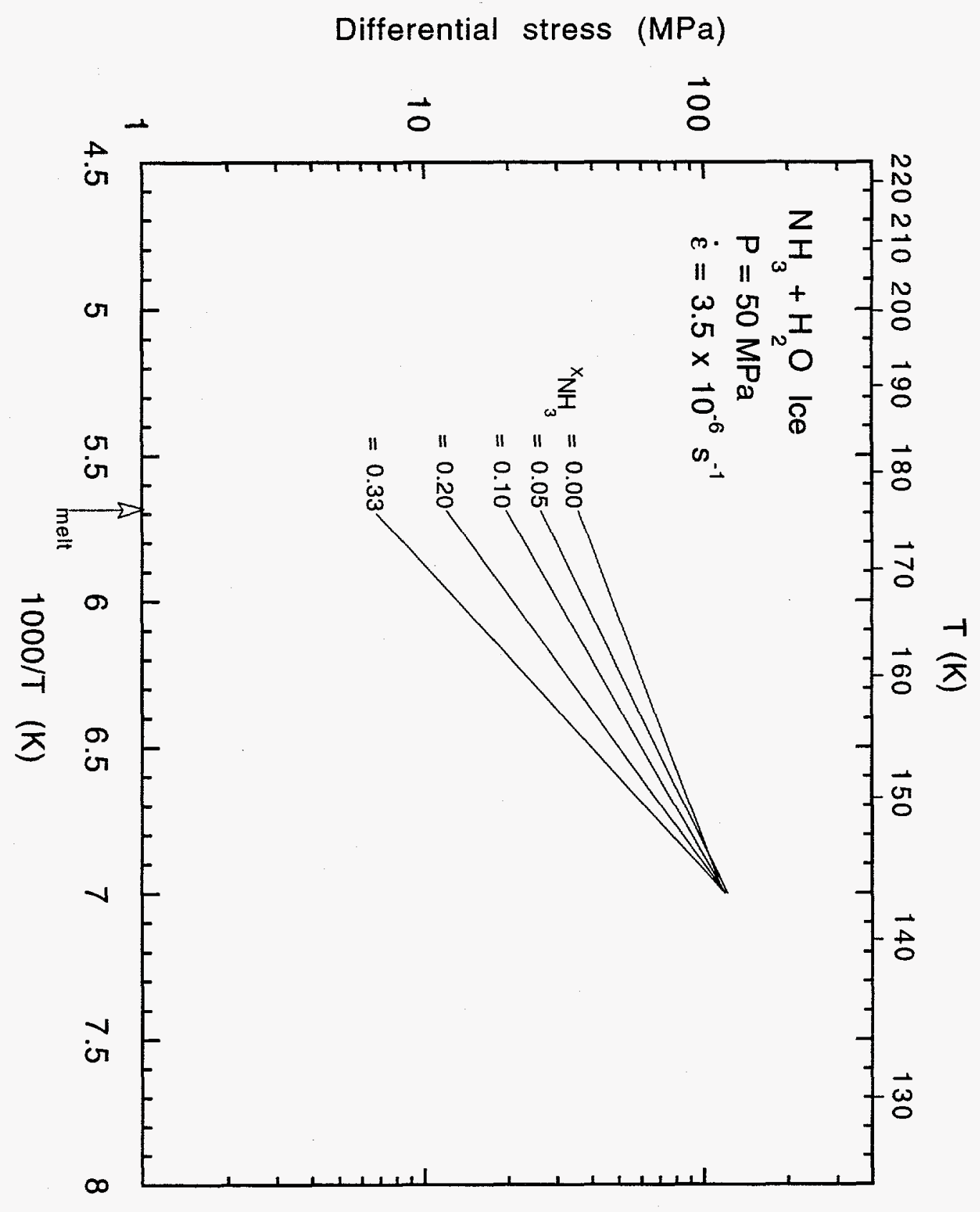

FIGURE 7 


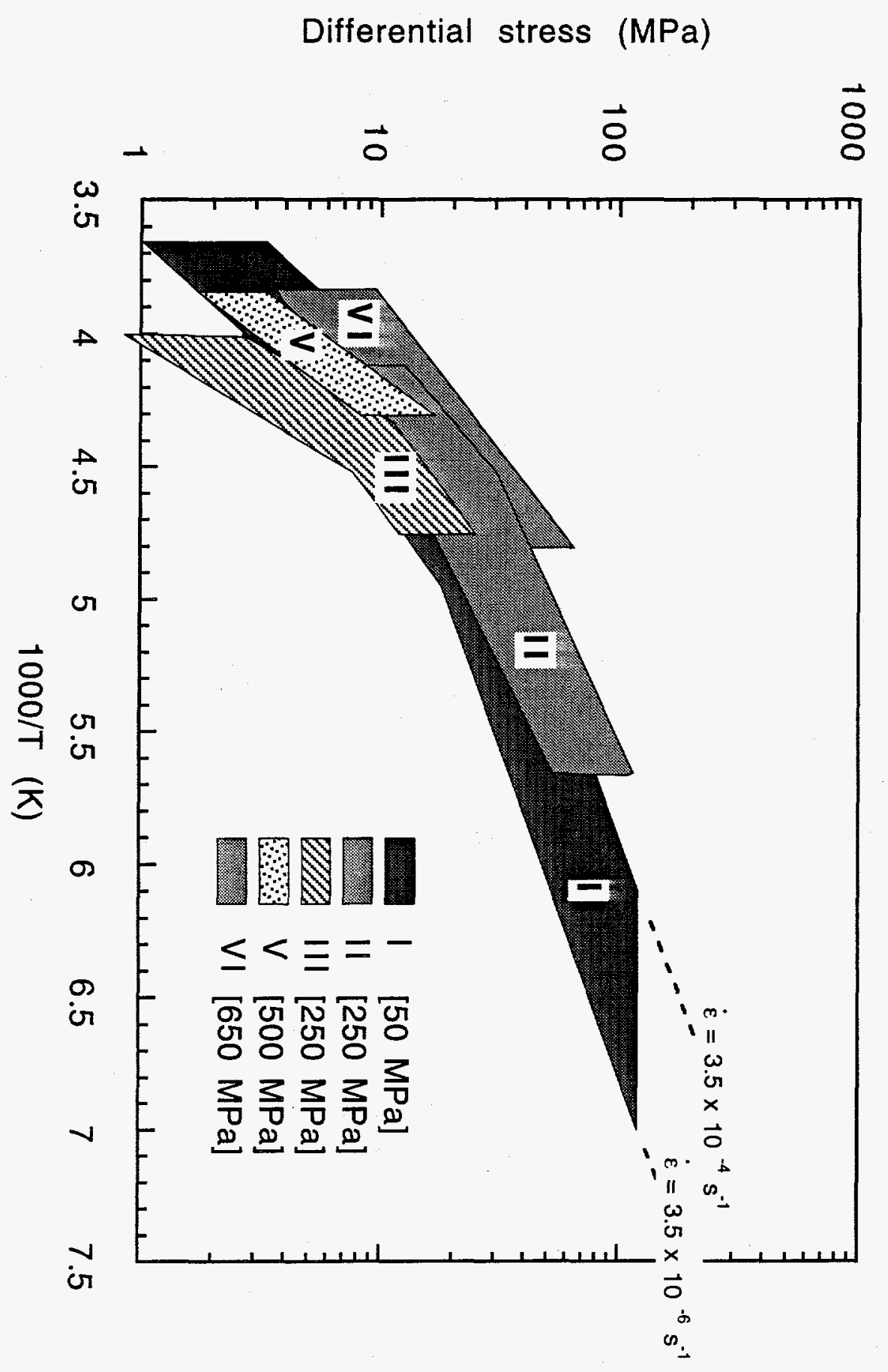

FIGURE 8 



$$
1
$$

